\title{
GESTÃO HUMANA PARA ORGANIZAÇÕES SAUDÁVEIS
}

\author{
Inara Antunes Vieira Willerding, Dra. ${ }^{\mathbf{~}}$ \\ Juliano Keller Alvez, Me. ${ }^{2}$; \\ Édis Mafra Lapolli ${ }^{3}$
}

\begin{abstract}
: this research aims to understand what healthy organizations are focused on human management. To this end, a descriptive and exploratory study was carried out, with a qualitative approach, in which the data collected were through learning developed in a Programmed Training Activity (AFP) carried out by the Graduate Program in Engineering and Knowledge Management ( PPGEGC) of the Federal University of Santa Catarina (UFSC), located in the south of Brazil, with 19 students from different professional areas. Data analysis demonstrated the importance of a management focused on human management so that the management of human capital, through its behavior, provides a healthy environment, favorable to collaboration, cooperation, commitment, engagement and motivation, contributing assertively to the success of organizations.
\end{abstract}

Keywords: Human Management; Healthy Organization; Human capital.

Resumo: esta pesquisa visa compreender o que são organizações saudáveis com foco na gestão humana. Para tal, realizou-se um estudo descritivo e exploratório, com abordagem qualitativa, em que os dados coletados foram por meio do aprendizado desenvolvido em uma Atividade de Formação Programada (AFP) realizada pelo Programa de Pós-Graduação em Engenharia e Gestão do Conhecimento (PPGEGC) da Universidade Federal de Santa Catarina (UFSC), localizada na região sul do Brasil, com 19 alunos de diferentes áreas de atuação profissional. A análise dos dados demonstrou a importância de uma gestão focada na gestão humana para que

\footnotetext{
${ }^{1}$ Programa de Pós-Graduação em Engenharia e Gestão do Conhecimento - Universidade Federal de Santa Catarina (UFSC) Florianópolis - Brasil. Correo electrónico: inara.antunes@gmail.com

${ }^{2}$ Programa de Pós-Graduação em Engenharia e Gestão do Conhecimento - Universidade Federal de Santa Catarina (UFSC) Florianópolis - Brasil. Correo electrónico: juliano@ ceteg.net.br

${ }^{3}$ Programa de Pós-Graduação em Engenharia e Gestão do Conhecimento - Universidade Federal de Santa Catarina (UFSC) Florianópolis - Brasil. Correo electrónico: edispandiom@gmail.com
} 
o gerenciamento do capital humano, por meio de seu comportamento, propicie um ambiente saudável, favorável a colaboração, cooperação, comprometimento, engajamento e motivação contribuindo de forma assertiva para o sucesso das organizações.

Palavras-chave: Gestão Humana; Organização Saudável; Capital Humano.

\section{INTRODUÇÃO}

À medida que as organizações se desenvolvem e evoluem, novos desafios surgem para que elas se mantenham sustentáveis. Novos termos surgem, os antigos são redefinidos e os estudos passam a trazer achados que tem significância para o dia-a-dia das organizações.

Nesse sentido, os conceitos de "Gestão Humana" e "Organizações Saudáveis" aparecem nos estudos e na rotinas das organizações com alguma frequência, sem que o sentido dessas duas expressões seja estratificado e estudado de forma mais aprofundada, a ponto de obter-se um entendimento convergente àquele que os autores que trabalham esses temas já tenham estabelecido.

É sabido, logo em um primeiro momento, que gestão humana não é sinônimo de gestão de pessoas e, da mesma forma, que organização saudável não é aquela que somente obtém lucro em suas operações ou trata o meio ambiente de forma adequada. Trata-se de expressões que possuem conceitos muito mais profundos e importantes para a gestão das organizações, visto que no centro do entendimento de ambas, estão temas que podem potencializar o sucesso ou insucesso de uma determinada companhia ou negócio.

A gestão humana nos parece fundamental para que a organização capte e retenha talentos que façam a diferença em seus resultados, e ainda, engaje e reúna pessoas em um propósito maior. Já as organizações saudáveis representam o estado da arte da gestão, pois possuem características específicas que as tornam diferentes e com melhor desempenho que as demais organizações.

Por esse motivo, pretende-se com este artigo, compreender o que são organizações saudáveis com foco na gestão humana, oportunizando às organizações e à comunidade acadêmica a possibilidade de refletir sobre essas duas expressões com maior vigor, provocando 
de forma especial as organizações a atuarem com posturas, práticas e comportamentos alinhadas aos conceitos aqui apresentados.

\section{EMBASAMENTO TEÓRICO}

Este tópico oferece suporte teórico a esta pesquisa, pois de acordo com Triviños (2002, p. 104) não é possível interpretar, explicar e compreender a realidade sem um referencial teórico".

\subsection{GESTÃO HUMANA}

A complexidade e a incerteza no mundo dos negócios pela dinamicidade decorrente da globalozação eda inovação tecnológica fazem com que as organizações passem a buscar talentos com competências que as diferenciem das demais, sendo estratégias importantes para o seu desenvolvimento e crescimento.

Assim percebe-se que um dos pilares das estratégias organizacionais está no seu capital humano, fazendo-se necessário melhores práticas e entender a sua contribuição para a eficácia da organização. Dessa forma, segundo Gómez e Hernández (2011), se faz necessário gerir o capital humano existente no ambiente organizacional, alinhando os interesses, as metas e objetivos da organização, com o bem-estar de seus profissionais, sendo esse o papel atual da gestão humana.

A gestão humana para Seiffert (2002, p. 65) refere-se a "um conjunto de políticas, processos, programas e ações para selecionar, aplicar, manter, desenvolver e monitorar seres humanos para atingir objetivos organizacionais, através de suas capacidades, habilidades, motivações e comprometimento", e ainda, "a gestão humana tem como principal foco de ação a motivação e comprometimento das pessoas para ampliar e aplicar suas capacidades e habilidades geradoras de riqueza".

Tendo a Gestão Humana como fator estratégico, Goméz e Hernández (2011) afirmam que desenvolver as melhores práticas se torna essencial para aprendizagem organizacional, pois 
a formação dos profissionais é uma forma de desenvolver habilidades objetivando torná-los mais produtivos e inovadores agregando assim, alor à empresa.

Diante dessa perpectiva, Solarte, Rodríguez e León (2013) dizem que a Gestão Humana é essencial para a excelência organizacional de forma estratégica pelos seus processos, pelo uso adequado dos talentos de seus profissionais e pela capacidade de harmonizar equipes para maior eficiência na conquista de seus objetivos, definindo assim, o capital humano como a melhor estratégia de crescimento, desempenho, produtividade e competitividade da organização.

Corroborando, Saldarriaga (2014) diz que a Gestão Humana e suas práticas, devem ser tratadas como essencial e inclusivo nas organizações com a função de resgate do ser humano, tanto na gestão, quanto nas práticas de trabalho.

Consolidando a fala de Saldarriaga (2014), Martínez, Oviedo e Luna (2015) afirmam que essas práticas amparam a melhoria das condições de trabalho dos profissionais, trazendo benefícios não só para o colaborador, mas para as organizações também. Pois, “[...] ações de gestão humana são implantadas para possibilitar que a satisfação das necessidades humanas combine-se com as necessidades da empresa" (Seiffert, 2002, p. 212).

Com os olhares voltados para as relações humanas, o ser humano passa a ser o protagonista, "começa-se à entender que o colaborador faz parte do processo, e surgem novos estilos de liderança, a preocupação com o grupo, e as situações que os norteiam, como a necessidade de administrar os conflitos" (Lamoglia et. al., 2015, p. 5).

Tendo o ser humano como protagônico, tem-se a concepção de ser ativo, capaz de,

transformar as circunstâncias que os rodeiam de maneira consciente, digno de respeito e participação, baseado em princípios de equidade, liberdade, responsabilidade: com capacidade de decisão, autonomia, aprendizado e desenvolvimento, sendo parte da organização como um sujeito criativo que contribui para o alcance da missão e visão organizacional, ao mesmo tempo em que busca sua realização pessoal (Patino \& Arbelaz, 2016, p. 2).

Assim, pode-se considerar o ser humano ativo e afetivo, em que "a afetividade, a convivência e a humanização são indissociáveis e a gestão humanizada tem por objetivo levar a empresa a adotar um novo paradigma, fazendo do homem o sujeito de sua atenção e dedicação, olhando a vida na sua transcendência com foco no bem coletivo", e também de 
desenvolvimento pessoal, com senso de coletividade, visão sistêmica, transparência, autonomia, responsabilidade e diversidade (De Mario, 2019, p. 3).

Conde, Vargas e Moreno (2018) trazem em sua pesquisa as práticas de gestão humana que dentre outras, oferece:

- Oportunidade de crescimento, treinamento, qualificação e desenvolvimento;

- Oportunidades de carreira;

- Avaliação de desempenho, promoções e remuneração justa;

- Descentralização do poder decisório;

- Olhar sistêmico;

- Igualdade de gênero;

- Melhora na qualidade de vida no trabalho.

A gestão humana tem entre seus principais desafios o alinhamento entre o indivíduo com a estratégia organizacional, dessa forma,

[...] deve reconhecer as particularidades dos seres humanos, suas características pessoais, psicológicas e sociais, ajudar a gerar os melhores relacionamentos entre os membros da organização e proporcionar a eles as melhores condições para o desenvolvimento de seu trabalho. [...] implica a concepção de um ser humano atuante, capaz de transformar o ambiente organizacional e de lhe dar um toque pessoal para contribuir para a consecução de seus objetivos (Ríos, 2014, p. 231).

Essa transformação deve transcender as práticas funcionais dos profissionais, reconhecendo as necessidades e oferecendo ferramentas para motivar e capacitar com o objetivo de qualificar, impulsionando o desenvolvimento de talentos, encorajando os valores da organização, o respeito e o trabalho em equipe, estimulando as atitudes e o comportamento do profissional em relação ao alcance das estratégias competitivas da organização, respondendo a problemas críticos enfrentados com criatividade e inovação

\subsection{ORGANIZAÇÕES SAUDÁVEIS}


Vergara e Branco, em 2001, já vinham explanando a necessidade e a viabilidade das organizações serem humanizadas. Os autores entendem que uma organização humanizada possui seu foco no colaborador e no ambiente de trabalho, agregando valores organizacionais, não somente lucrativos.

Realiza ações que, no âmbito interno, promovem a melhoria na qualidade de vida e de trabalho, visam à construção de relações mais democráticas e justas, mitigam as desigualdades e diferenças de raça, sexo ou credo, além de contribuírem para o desenvolvimento das pessoas sob os aspectos físico, emocional, intelectual e espiritual. Ao focalizar o ambiente, essas ações buscam a eliminação de desequilíbrios ecológicos, a superação de injustiças sociais, o apoio a atividades comunitárias, enfim, o que se convencionou chamar de exercício da cidadania corporativa (Vergara \& Branco, 2001, p. 21).

Esse exercício da cidadania corporativa, pode-se dizer que refere-se a uma visão sistêmica da organização, em que busca o equilíbrio e o alinhamento dos interesses corporativos com a de seus colaboradores, que para Csikszentmihalyi (2004), refere-se aos princípios básicos de uma organização saudável, em que a relação indivíduo e organização está pautada na harmonia entre qualidade de vida, bem-estar, felicidade, produtividade com a qualidade e rentabilidade.

Para Barrios e Paravic (2006) organizações saudáveis refere-se ao ambiente organizacional favorável ao bem-estar dos profissionais, visando não somente o ambiente físico, mas as interrelações, a organização e a saúde emocional. Assim, pode-se dizer que as organizações saudáveis são aquelas que "reconhecem que as necessidades dos funcionários e da organização são mutuamente interdependentes e que se esforçam para atender à agenda dupla de qualidade de vida dos funcionários e sucesso e sustentabilidade organizacional" (Lewis et.al, 2011, p. 65). E ainda,

um local de trabalho vibrante é realmente a peça central do modelo [de organizações saudáveis], porque este é o ambiente no qual as pessoas trabalham dia após dia. Locais de trabalho vibrantes fazem mais do que envolver os funcionários, cultivam um senso de inspiração pessoal sobre o trabalho, pois um colaborador engajado é satisfeito e efiel, inspirado é mais do que isso, buscam ativamente maneiras de desenvolver e usar habilidades e conhecimento para promover os objetivos corporativos (Lowe, 2012, p. 21). 
Essa vibrações “promovem negócios saudáveis baseando-se em uma conexão estreita entre bem-estar dos colaboradores e organizacional e funcionamento eficaz" proveniente de uma gestão saudável (Di Fabio et al, 2016, p. 2). Essa forma de gerir permite que seus colaboradores coloquem em prática suas capacidades, pois:

precisam de relacionamentos, recursos e sistemas que lhes permitem colaborar, quando os trabalhadores colaboram, a soma torna-se maior do que as partes: equipes e organizações desenvolvem capacidades de desempenho, inovação e criatividade que ultrapassa em muito o que cada membro traz para seu trabalho e para organização, seus clientes e a sociedade (Lowe, 2012, p. 41).

Diante do exposto, Acosta et al (2015, p. 325) afirmam que “[ ...] organização saudável significa ter uma visão ampla de seu ambiente, onde aspectos como as características dos sistemas de trabalho, valores culturais e clima organizacional são levados em consideração." Essa forma ampla de enxergar a organização refere-se a uma visão sistêmica que realizam ações sistemáticas, planejadas e proativas para melhorar os processos e resultados de seus colaboradores e da organização como um todo (Salanova et al., 2019).

Nessa perspectiva, as organizações saudáveis permitem:

trilhar um caminho positivo para a produtividade e a sustentabilidade da organização e do trabalhador por meio de um enfoque salutogênico, no qual saúde e segurança se tornam valores estratégicos autênticos pra toda a comunidade organizacional. Este caminho aponta para práticas saudáveis que geralmente constituem organizações saudáveis e que buscam se alinhar ao conceito de trabalho decente e da dignidade humana (Guimarães et al., 2018, p. 511).

Uma gestão pautada no respeito, na potencialidade de cada indivíduo, valores e vitalidade dos profissionais, com foco nas interrelações, interações positivas e realizações por meio da coletividade, geram trabalhos com significado e pertencimento social (Guimarães et al., 2018). Para isso, "há de se manter atenção às constantes transformações sociais, dos estilos de gestão, de organização do trabalho (no Brasil, por exemplo, a nova legislação trabalhista) e de novas tecnologias nesses ambientes" (Guimarães et al., 2018, p. 512). 
Diante dessas colocações, “[...] a organização saudável é, portanto, um local de trabalho que atende à dupla agenda de alcançar eficiência e eficácia organizacional e atender às necessidades de apoio, motivação e incentivo dos funcionários (Billquist, Szücs \& BäckWiklund, 2012, p. 148).

\section{METODOLOGIA}

De acordo com o objetivo a ser alcançado nessa pesquisa: compreender o que são organizações saudáveis com foco na gestão humana, e para a obtenção dos resultados, essa pesquisa se classifica como bibliográfica pela busca de evidências em dados já publicados, visando subsidiar a elaboração da revisão bibliográfica e a análise de resultados. Classifica-se também como descritiva, uma vez que os dados gerados foram analisados, apresentando as situações e demais características sobre a investigação proposta e exploratória por entender que trata-se de uma pesquisa que gera novos conhecimentos. Também considera-se uma pesquisa com abordagem do tipo aplicada pois "é voltada à aquisição de conhecimentos com vistas à aplicação numa situação específica" (Gil, 2018, p. 26).

A coleta de dados se deu em uma Atividade de Formação Programada (AFP) realizada pelo Programa de Pós-Graduação em Engenharia e Gestão do Conhecimento (PPGEGC) da Universidade Federal de Santa Catarina (UFSC), localizada na região sul do Brasil, com 19 alunos de diferentes áreas de atuação profissional.

Após a apresentação e discussão da atividade em sala, os dados foram coletados na versão online disponibilizado na plataforma moodle, Ambiente Virtual de Aprendizagem (AVA), compondo um arcabouço de 14 respostas, haja vista que alguns participantes realizaram a atividade em dupla.

\section{APRESENTAÇÃO E ANÁLISE DOS RESULTADOS}

Durante os encontros da Atividade de Formação Programada (AFP) sob o título Gestão Humana para Organizações Saudáveis, em uma das atividades previstas previamente no planejamento, foi solicitado aos participantes que fizessem uma busca dos conceitos de "Gestão 
Humana" e de "Organizações Saudáveis" e que sugerissem o "seu” conceito próprio sobre essas duas expressões.

Como método na AFP, utilizou-se a sala de aula invertida (flipped classroom), considerada uma metodologia ativa por propiciar "o aprender a aprender de formas diferentes o mesmo conteúdo, transformando o processo de ensino-aprendizagem significativo para os estudantes" (Beux, 2019, p. 3).

Conforme Valente (2014, p. 85),

a sala de aula invertida é uma modalidade de e-learning na qual o conteúdo e as instruções são estudados on-line antes de o aluno frequentar a sala de aula, que agora passa a ser o local para trabalhar os conteúdos já estudados, realizando atividades práticas como resolução de problemas e projetos, discussão em grupo, laboratórios etc.

Uma prática referente a “uma migração do 'ensinar' para o 'aprender', o desvio do foco do docente para o aluno, que assume a corresponsabilidade pelo seu aprendizado" (Souza, Iglesias \& Pazin-Filho, 2014, p. 285). E que conforme Willerding e Lapolli (2020, p. 190) "têm o aluno como foco, em que ele é o centro do processo de ensino e de aprendizagem e como ele está vendo o mundo, onde sai da função de expectador e assume o papel de protagonista".

Como planejado, os participantes da pesquisa realizaram uma busca bibliográfica com o termo "organizações saudáveis", trazendo para a sala de aula o seu próprio conceito pautado na pesquisa bibliográfica, os quais são apresentados participantes no Quadro 1:

Quadro 1 - Conceitos de “Organizações Saudáveis” desenvolvidos pelos participantes

\begin{tabular}{|l|c|}
\hline \multicolumn{1}{|c|}{ Conceito dos participantes } & Responsável(eis) \\
\hline $\begin{array}{l}\text { São aquelas que praticam valores éticos, morais, equidade, } \\
\text { igualdade e aprendizado contínuo. Constroem relações saudáveis } \\
\text { em todos os níveis e promovem o desenvolvimento sustentável de } \\
\text { seus integrantes baseado em propósito. }\end{array}$ & Aline Debize de Fraga \\
\hline $\begin{array}{l}\text { São organizações que consideram o bem-estar das pessoas tão } \\
\text { importante quanto os resultados organizacionais. São organizações } \\
\text { que buscam a melhoria dos resultados através da promoção do bem- } \\
\text { estar individual. }\end{array}$ & $\begin{array}{c}\text { Lapolli e Juan Enrique } \\
\text { Pereira Adan }\end{array}$ \\
\hline $\begin{array}{l}\text { Funcionam como um sistema, olhando o todo e cada um do mesmo } \\
\text { modo, com foco na saúde dos funcionários, da empresa e do meio, } \\
\text { garantindo assim a sua sustentabilidade. }\end{array}$ & $\begin{array}{c}\text { Melissa Ribeiro do } \\
\text { Amaral e Denise } \\
\text { Terezinha Almeida } \\
\text { Marcon }\end{array}$ \\
\hline
\end{tabular}


São aquelas que buscam atingir seus objetivos organizacionais sem descuidar da qualidade de vida no trabalho e bem estar de seus trabalhadores, oferecendo oportunidades de crescimento profissional e ações visando a melhoria e sustentabilidade da comunidade na qual está inserida, de forma a promover o crescimento e bem-estar de todos: organização, trabalhadores e comunidade.

Caracteriza-se por tornar estratégica a dualidade dos interesses da organização e de seus colaboradores, promovendo uma harmonia capaz de gerar vantagem competitiva para enfrentar os paradigmas do mercado.

Promovem o bem-estar interno e externo à organização, por meio de práticas sistêmicas não lineares que consideram o todo, e asseguram as particularidades, especificidades e necessidades de cada ator que a compõe.

Ao observar os conceitos dos participantes para o termo "Organizações Saudáveis" é possível perceber que a satisfação de todas as partes interessadas é uma característica marcante desse tipo de organização, ou seja, desenvolver relações de excelência com clientes, colaboradores, fornecedores, sócios, comunidade e governo.

Termos como "qualidade de vida no trabalho" ou "bem estar dos trabalhadores" também apareceram com frequência nos conceitos, o que parece ser um comportamento importante para as organizações saudáveis, no sentido de cuidar das pessoas como um todo e não apenas no conceito estreito de funcionário.

O equilíbrio na busca por resultados organizacionais e na valorização das pessoas foi apresentado em alguns conceitos, visto ser um desafio de grande parte das empresas, pois muitas vezes os objetivos do colaborador divergem dos objetivos da companhia.

De igual forma, os participantes realizaram busca bibliográfica com o termo "gestão humana" e trouxeram para a sala de aula o seu próprio conceito baseado na pesquisa, os quais são apresentados o Quadro 2:

Quadro 2 - Conceitos de "Gestão Humana" desenvolvidos pelos participantes

\begin{tabular}{|l|c|}
\hline \multicolumn{1}{|c|}{ Conceito do aluno } & $\begin{array}{c}\text { Aluno(s) } \\
\text { responsável(eis) }\end{array}$ \\
\hline $\begin{array}{l}\text { Consiste em olhar para o "ser" com empatia, buscando conhecer } \\
\text { seus sentimentos e necessidades, proporcionando oportunidades de } \\
\text { desenvolvimento, crescimento, com justiça, equidade, acreditando }\end{array}$ & Aline Debize de Fraga \\
\hline
\end{tabular}


no potencial de melhoria e contribuição. Compreender a complexidade humana e como se relaciona com a organização, promovendo alinhamento de valores, afetividade, emoções positivas e incentivo justo ao alcance de resultados coordenados com os objetivos organizacionais.

É um processo de aprimoramento e busca de uma relação mais humanitária entre todos os atores sociais envolvidos nas organizações.

Visa conciliar os propósitos da organização com a qualidade de vida e as competências dos colaboradores em uma perspectiva de ganho mútuo.

A gestão humana observa as particularidades do indivíduo, identifica necessidades, disponibiliza ferramentas, proporciona o desenvolvimento sistêmico da organização, consolida os valores da empresa, potencializa as competências do capital humano e alinha os interesses dos steakholders, tendo como finalidade uma organização saudável.

É a humanização das relações, incluindo valores como dignidade, liberdade, identidade, respeito, crescimento, aceitação e diálogo contínuo, onde o indivíduo é compreendido como um ser integral e em constante evolução, que deve ser ouvido e incluído nos processos decisórios, de modo a desenvolver o sentimento de pertencimento e comprometimento, com ética e responsabilidade para com os rumos e resultados da organização

Consiste em práticas que utilizam o fator humano como estratégia de crescimento organizacional, priorizando a relação "colaboradororganização", na busca dos objetivos organizacionais, contribuindo com maior desempenho, produtividade e competitividade frente ao mercado.

Alix Ribeiro da Silva e Juliana Cidrack Freire do Vale

Joiceli Rossoni Lapolli e Juan Enrique Pereira Adan

Melissa Ribeiro do Amaral e Denise

Terezinha Almeida Marcon

Waldoir Valentim

Gomes Junior e

Felipe Kupka

Feliciano

Ao analisar os conceitos dos participantes para o termo "Gestão Humana" foi possível identificar que o "equilíbrio entre a busca dos resultados das organizações e no respeito às particularidades do indívíduo" foi a essência percebida na maioria dos textos.

De igual modo, percebe-se que organizações empáticas, que realizam ações para a qualidade de vida e desenvolvem as pessoas não apenas em suas competências técnicas, mas como um todo, também podem ser consideradas praticantes da gestão humana.

Outro aspecto importante que está intrínseco em cada conceito é que as necessidades das pessoas mudaram ao longo do tempo. No passado, elas opinavam menos, participavam pouco e o objetivo do trabalho era meramente o sustento da casa ou da família. Com o passar do tempo, as pessoas esperam mais: desejam ser respeitadas, ouvidas e bem tratadas. Orgulham- 
se ou não da organização da qual fazem parte, de acordo com o propósito e a identidade que a empresa consegue comunicar ao colaborador.

Pensar no indivíduo, importar-se com ele, com sua forma de vida e seus anseios, mesmo em meio a todos os desafios de competitividade, agilidade e de busca de resultados que as organizações enfrentam, torna-se um dos focos centrais da gestão humana.

\section{CONSIDERAÇÕES FINAIS}

As expressões "Gestão Humana" e "Organizações Saudáveis" são de absoluta relevância para as organizações.

Na busca do entendimento do termo "Gestão Humana" percebeu-se o clamor pelo equilíbrio entre a busca de resultados e o respeito às necessidades das pessoas, as quais esperam um tratamento igualitário, respeitoso e que considere seus anseios pessoais e de desenvolvimento profissional, sem que se perca de vista a necessidade de se gerar resultados positivos na produtividade, lucratividade e outros indicadores importantes na medição de desempenho organizacional.

Sabe-se que as pessoas são essenciais para a escalada de competitividade das organizações e uma gestão humana colabora para compreender necessidades, planejar e executar ações convergentes àquilo que as pessoas esperam, visando a criação de um clima de trabalho diferenciado e que potencialize os predicados pessoais e profissionais de cada um.

Quando tratamos de "Organizações Saudáveis", percebemos que os conceitos convergem para o tratamento de todas as partes interessadas (stakeholders) com excelência, em que considera-se a satisfação de clientes, colaboradores, comunidade, fornecedores e sócios, dentre outros. Com esta postura, a organização estará agindo para que seus resultados corporativos sejam cada vez melhores, uma vez que a postura dela com as partes interessadas conduzirá a ganhos significativos ao longo do tempo.

Certamente, os dois conceitos aqui apresentados não são finitos e há muito a ser desenvolvido em novas investigações, visando o entendimento e a prática diária desses termos nas organizações. É possível que em uma pesquisa mais ampla com gestores organizacionais, 
novos conceitos surjam e enriqueçam ainda mais o que foi gerado neste momento por este estudo.

\section{AGRADECIMENTOS}

O presente trabalho foi realizado com apoio da Coordenação de Aperfeiçoamento de Pessoal de Nível Superior - Brasil (CAPES) - Código de Financiamento 001.

\section{REFERÊNCIAS}

Acosta, H., Cruz-Ortiz, V., Salanova, M. \& Llorens, S. (2015). Healthy organization: analysing its meaning based on the HERO Model / Organizaciones saludables: analizando su significado desde el Modelo HERO. Revista de Psicología Social (vol. 30, n. 2, pp. 323350)

Barrios, S. \& Paravic, T. (2006). Promoção saúde e um ambiente de trabalho saudável. Revista Latino americana Enfermagem (vol. 1, n. 14, pp. 136-141).

Billquist, L., Szücs, S. \& Bäck-Wiklund M. (2012). Care: A Challenge To Healthy Organizations? A Case Study Of A Hospital Department. Sociologia, Problemas e Práticas (vol. 68, pp. 147-167)

Conde, E. B., Vargas, S. M. Z. \& Moreno, O. R. M. (2018, Julio/Diciembre). Prácticas de gestión humana. Observación y análisis en las pequeñas empresas manufactureras del corredor industrial de Boyacá. revista Encuentros, Vol. 16, pp. 157-168. DOI: http://dx.doi.org/10.15665/encuent.v16i02.1569.

Csikszentmihalyi, M. (2004). Gestão qualificada: A conexão entre felicidade e negócios. Porto Alegre: Bookmam.

De Mario, M. (2019). Gestão Humanizada: afetividade, convivência e humanização. Idealizador do IBEM. Edição Kindle.

Di Fabio, A., Giannini, M., Loscalzo, Y., Palazzeschi, L., Bucci, O., Guazzini, A. \& Gori, A. (2016) The Challenge of Fostering Healthy Organizations: An Empirical Study on the Role of Workplace Relational Civility in Acceptance of Change and Well-Being. Frontiers in Psychology. (vol. 7, n. 1748, pp. 1-9).

Gil, A. C. (2018). Métodos e Técnicas de Pesquisa Social. 6. ed. São Paulo, Atlas.

Gómez, C. A. T. \& Hernández, G. C. (2001, Julio/Diciembre). ¿Agregan valor las áreas de gestión humana a las organizaciones del sector industrial de Duitama y Sogamoso (Boyacá)? Entramado. Vol.7 No. 2, pp. 54-71. 
Guimarães, L. A. M., Oliveira, F. F. de., Massuda Junior, J. \& Rebolo, F. (2018, out-dez). Resenha - Em busca de organizações saudáveis. Revista Psicologia: Organizações e Trabalho, 18(4) pp. 511-513. DOI: 10.17652/rpot/2018.4.15202.

Lamoglia, C. V. A., Silva, E. M. V. da., Machado, V. F., Olímpio, M. M. \& Souza, F. P. (2015). Relações Umanizadas no Contexto empresaral: uma realidade em construção. XII SEGeT - AEDB - XII Simpósio de Excelência em Gestão e Tecnologia. Rio de Janeiro - Resende, pp. 1-16.

Lewis S., Doorne-Huiskes A., Redai D. \& Barroso M. (2011). Healthy Organizations. In: BäckWiklund M., Van Der Lippe T., Den Dulk L., Doorne-Huiskes A. (eds) Quality of Life and Work in Europe. Palgrave Macmillan, London.

Martinez-buelvas, L., Oviedo-Trespalacios, O. \& Luna-Amaya, C. (2015). Impact of working conditions on the quality of working life: Case manufacturing sector colombian Caribbean Region. Dyna rev.fac.nac.minas [online].(vol.82, n.194, pp.194-203).

Patino, J. D. P. \& Arbelaz, I. C. L. (2016, fev.). Gestión humana de orientación analítica: un camino para la responsabilización. Revista de Administração de Empresas (vol. 56, n. 1, pp. 101-113).

Ríos, J. G. S. (2014, ago.). La concepción del sujeto en la administración: una mirada desde la gestión humana (vol. 14, n. 1, pp. 1-325). Medellín-Colombia.

Saldarriaga, J. (2014, aug). A concepção do sujeito na administração: um olhar a partir da gestão humana (14, pp. 223-237) USB.

Seiffert, P. Q. (2002). Modelo de Gestão Humana para empresas intensivas em capital intelectual: um ensaio na EMBRAER S.A. Florianópolis, 2002. Tese (Doutorado em Engenharia de Produção) Programa de Pós-graduação em Engenharia de Produção. UFSC, Florianópolis.

Solarte, M. G., Rodríguez, A. R. A. \& León, J. D. P. (2013, Julio/Septiembre). Prácticas de Responsabilidad Social Empresarial desde las áreas funcionales de Gestión Humana: análisis de resultados en cuatro empresas del suroccidente colombiano. Revista INNOVAR (vol. 23, n. 49, pp. 83- 100) Bogotá.

Souza, C. S., Iglesias, A. G. \& Pazin-Filho, A. (2014). Estratégias inovadoras para métodos de ensino tradicionais - aspectos gerais. Medicina (vol. 47, n. 3, pp. 284-2920.

Triviños, A. N. S. (2020). Introdução à pesquisa em ciências sociais: a pesquisa qualitativa em educação. São Paulo: Atlas.

Valente, J. A. (2014). Blended learning e as mudanças no Ensino Superior: a proposta da sala de aula invertida. Educar em Revista, (n. 4, pp. 79-97) Curitiba, Edição Especial.

Vergara, S. C. \& Branco, P. D. (2001, Abr./Jun.). Empresa Humanizada: a organização necessária e possível. RAE - Revista de Administração de Empresas. São Paulo. Vol. 41, n. 2, pp. 20-30.

Willerding, I. A. V. \& Lapolli, E. M. (2020). Educação empreendedora: o uso da criatividade na formação do profissional do século XXI. In: Teixeira, C. S., Lebler, C. D. C., Souza,

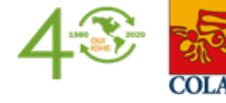


Ciudad del Saber, Panamá

X Congreso internacional de conocimiento e innovación

M. V. Educação fora da caixa: tendências internacionais e perspectivas sobre a inovação na educação. São Paulo: Blucher (vol. 5, pp. 181-195). 